\title{
MHealth and perceived quality of care delivery: a conceptual model and validation
}

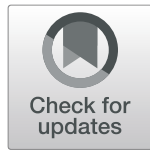

Yvonne O'Connor ${ }^{*^{*}}$ (D) Pavel Andreev ${ }^{2}$ and Philip O'Reilly ${ }^{1}$

\begin{abstract}
Background: The objective of this research is to examine, conceptualize, and empirically validate a model of mobile health (mHealth) impacts on physicians' perceived quality of care delivery (PQoC).

Methods: Observational quasi-experimental one group posttest-only design was implemented through the empirical testing of the conceptual model with nine hypotheses related to the association of task and technology characteristics, self-efficacy, m-health utilization, task-technology fit (TTF), and their relationships with PQoC. Primary data was collected over a four-month period from acute care physicians in The Ottawa Hospital, Ontario, Canada. The self-reported data was collected by employing a survey and distributed through the internal hospital channels to physicians who adopted iPads for their daily activities.
\end{abstract}

Results: Physicians' PQoC was found to be positively affected by the level of mHealth utilization and TTF, while the magnitude of the TTF direct effect was two times stronger than utilization. Additionally, self-efficacy has the highest direct and total effect on mHealth utilization; in the formation of TTF, technological characteristics dominate followed by task characteristics.

Conclusion: To date, the impact of utilized mHealth on PQoC has neither been richly theorized nor explored in depth. We address this gap in existing literature. Realizing how an organization can improve TTF will lead to better PQoC.

Keywords: Mobile systems, Post-implementation, Information technology utilization, Quality of care

\section{Contributions to the literature}

- The use of mHealth and physicians' perceptions regarding their satisfaction and performance as it impacts upon quality of patient care is under investigated.

- A conceptual model is developed and empirically examined to understand the factors which impact physicians' perceived quality of care (PQoC) when employing mHealth artefacts when delivering healthcare services in a hospital setting.

- Our findings demonstrate that physicians using mHealth at the point-of-care enhances their PQoC a patient receives. These findings can be used to inform implementation strategies to increase the use of mHealth in hospitals.

\footnotetext{
* Correspondence: Y.OConnor@ucc.ie

${ }^{1}$ Department of Business Information Systems, University College Cork, O' Rahilly Building, Cork, Ireland

Full list of author information is available at the end of the article
}

\section{Background}

The mobile health (mHealth) market has experienced a significant growth since 2011, with the global mHealth market estimated to grow to about $\$ 60$ billion by 2020 . Lee [1] argued that that many hospitals are spending vast amount of money for implementing mHealth solutions and expecting that users (i.e. physicians) will employ the technology to improve the quality of care patients receive at the point-of-care. Concurrently, hospitals face increasing demands to participate in a wide range of quality improvement activities $[2,3]$ by streamlining their processes in order to deliver high quality and safe care $[4,5]$. Instant real-time access to data at the point-of-care is causing a paradigm shift in how physicians deliver healthcare, making services more streamlined and cost effective [6].

Despite the wide endorsement and support for the implementation of mHealth, Rahurkar [7] argue that empirical evidence surrounding the benefits of Information

(c) The Author(s). 2020 Open Access This article is distributed under the terms of the Creative Commons Attribution 4.0 International License (http://creativecommons.org/licenses/by/4.0/), which permits unrestricted use, distribution, and 
Technology (IT) in health care remains to be firmly established. The limited evidence for the performance of mHealth technologies may be attributable to a lack of appropriate evaluation frameworks [8-10], with Boudreaux et al., [11] arguing that there exists no evalutation method which is mHealth specific. While some attempts have been made by various researchers [12-16] to evaluate mHealth, this work has predominantly focused on reviewing and reporting the adoption of mHealth technologies at early phases of implementation. The lack of evaluation across the mHealth field, primarily in post-adoption stages of implementation, may be perceived as a major weakness of the mHealth domain $[9,10]$. Therefore, for mHealth to be truly valuable and have a positive impact on quality of healthcare delivery, the IT artefact must be utilized post-adoption [17, 18]. Furthermore, Goodhue and Thompson [19] argue that the capabilities of the IT must match the tasks that the user must perform. Serrano and Karahanna [20] argue that the role of the user in influencing successful task performance has not been explored in-depth within a healthcare setting. Indeed, there has been a dearth of research focused on exploring an mHealth specific evaluation framework $[11,18]$ in a post-adoptive scenario, specifically in a hospital setting [21].

\section{MHealth and quality of care delivery}

MHealth, for the purposes of this study, refers to the application of mobile device/s and medical/clinical application(s) run on the device by physicians in a hospital domain, for communication, collaboration, and coordination of the physician's healthcare delivery daily activities in hospital premises including diagnosis, treatment, and disease management (adapted from [22]).

It is purported [23] that based upon ones experiences with using a new technology and having new information to hand, an individual forms a post-adoptive perception, which may potentially deviate from prior beliefs. Indeed, they note that such deviation will both impact user's ongoing perceived usefulness of the technology and influence user satisfaction, which will influence one's intention to continue to utilize the technology. While mHealth has promised major benefits at the national, regional, community, and individual levels, insufficient attention has been paid to the perceived quality of care delivery (PQoC) which can be obtained from using technological artefacts [7, 24, 25]. Research has established that negative outcomes from IT utilization are possible $[24,26]$. Therefore, if the utilization of mHealth is perceived to potentially harm the quality of care a patient receives, then it is unsafe for delivering healthcare services [27].

Various indicators for quality of care exist; namely, structure, process, and outcome of care delivery [28].
Quality of care is often measured from two perspectives; perceived and actual [24, 29], across several potential stakeholders (e.g. patients, physicians, administrative staff). Actual quality of care "relates to the interaction between health-care providers and patients and the ways in which inputs from the health system are transformed into health outcomes" [30]. Conversely, PQoC, in this study, is the physicians' perceptions regarding their satisfaction and performance as it impacts upon quality of patient care. Examining actual quality of care is extremely difficult to attribute wholly to the IT artefact in a complex multifaceted environment and requires an unambiguous evidence base [30]. Furthermore, Serrano and Karahanna ( [20] p.616) purport that the impact of mHealth utilized by physicians on PQoC (consultation delivery, in the context of Serrano and Karahanna's paper), has not been explored in depth. Therefore, the research addresses this call for research and puts forward the research question of what are the impacts mHealth artefacts have on physicians' perceived quality of care delivery in a hospital setting. To answer this question, we defined two main research objectives: 1) to examine and conceptualize a model of mHealth impacts on PQoC and 2) empirically validate this model.

\section{A perceived quality of care delivery model: theoretical underpinnings and model development}

Task-Technology Fit (TTF) reveals the association between IT and individual performance and is based on the premise of 'fit'. 'Fit' has been widely utilized and is associated with performance. For example, MIT's 90's model is underpinned by the theory of fit $[31,32]$ and argues that fit contributes to high performance [31-33]. Optimal performance occurs only if there is a tight fit (alignment) among the domains of strategy, structure, management processes, individual roles and skills and technology [31].

TTF operates at an individual level of analysis with the position that IT is more likely to have a positive impact on individual performance and be utilized if the capabilities of the IT match the tasks that the user must perform [19, 34]. It consists of five constructs namely, task characteristics, technology characteristics, task-technology fit, performance impacts and utilization. TTF theory as a theoretical lens for understanding the performance of electronic health (eHealth) technologies has previously been explored [35]. Specifically, Chiasson et al. [35] answers the call for research by Furneauz [36] to understand the effect of user performance on utilization and the association between the use of effective technology and user performance. They found TTF to be a useful theory for exploring IT in healthcare and illustrated the positive association between use and performance. 
However, in the context of performance, the association between the utilization of a technology and the PQoC (as a performance outcome) has not been investigated. It is noted [4, 37] that there is scant empirical evidence of the impact which healthcare information systems have on the quality of care. Towards deriving an understanding of same, a conceptual model is developed to explore physicians' view which mHealth has on PQoC. Specifically, a conceptual model is developed (based on the technology to Performance chain model) to focus on PQoC as the dependent variable.

\section{Hypotheses development}

Researchers have found that mobile technologies impact performance of mobile workers and promote efficiency [38-41]. When IT artefacts are embedded within an individual's work practice, then they must facilitate the accomplishment of their work [42, 43]. System usage for this study is defined as the degree to which mHealth is incorporated into the users' (i.e. physicians) work processes or tasks. The concept of usage focuses on incorporation and comprises routine, feature and value-adding use [44]. Feature use refers to the extent to which physicians use the mHealth features/functionality to complete any given task (adapted from [45]). Routine use is important in this study as this research is examined in a post-adoptive scenario. Therefore, routine use refers to the extent to which a healthcare physician tends to use mHealth automatically (adapted from [46]). Finally, value-adding use is the extent by which physicians capture "the additional (none-core, non-automated and/or non-compulsory) use by the user conducted to enhance the output or impact" ( [44] p.6).

Studies show that mHealth usage by physicians assists with facilitating decision support and medication safety in terms of prescribing and dispensing at the point-ofcare $[47,48]$, thereby increasing diagnoses while decreasing missed diagnoses. Similarly, it was found [25] that hospital implementation of Health IT was positively associated with activities intended to improve patient care quality. Therefore, it is hypothesized that:

\section{Hypothesis 1}

Physician's perceive that the Quality of Care delivered to their patients is positively impacted by the mHealth's alignment with the task at hand (TTF).

\section{Hypothesis 2}

Physician's perceive that the Quality of Care delivered to patients is positively impacted by physician's utilization of mHealth.

\section{Hypothesis 3}

Physician's utilization of mHealth is positively impacted by its alignment to the task at hand.

While technology is typically aligned with organizational structures, it is not aligned with care coordination i.e. healthcare physician tasks [2]. Task characteristics, in this study, are defined broadly as the actions taken by physicians and include flexibility, protocol adherence, and time criticality $[2,18]$. Due to the complex nature associated with the delivery of healthcare services, flexibility in workflows is clinically pertinent. The flexibility of a process is its ability to deal with both foreseen and unforeseen change [49]. Patient care in most environments is by its very nature a mobile experience [50]. Common problems which arise in healthcare settings include unavailable medical information at the time of treatment, replication of test results, protocols not being followed and prescription of incorrect medications [2]. Therefore, we propose the following hypothesis:

\section{Hypothesis 4-1}

Physicians perceptions of Task Technology alignment will be positively impacted by Healthcare task characteristics.

\section{Hypothesis 4-2}

Healthcare task characteristics impacts mHealth utilization by physicians in a healthcare setting.

Research argues that technological resources are required for system usage [51]. Technology (i.e. mobile) characteristics refer to specific features, functionality, or usability of a technology that can affect its usage by target users [52]. It is argued that the implementation of any eHealth technology must live up to its fullest potential in real-world conditions and circumstance [18, 53], therefore having real world value. Existing research argues that physicians may be reluctant to utilize some IT technologies because they may fear it will not perform reliably or possess insufficient functionality for users to perform tasks. Therefore, we hypothesize:

\section{Hypothesis 5-1}

Physician perceptions of Task Technology alignment will be positively impacted by mHealth characteristics.

\section{Hypothesis 5-2}

The mHealth characteristics impact upon its use by physicians in a healthcare setting.

Research [54, 55] argues that self-efficacy tailored to an IT artefact is an important determinant of a variety of user perceptions of technology. As a result, selfefficacy has received considerable empirical support in a vast array of papers spanning both pre-and postadoption research studies. Self-efficacy is defined as the degree to which an individual's perceives their ability to 
utilize mHealth in the accomplishment of a task (adapted from [56]). Shaw and Manwani [57] found that physicians with high self-efficacy had greater potential to extensively use the vast array of features offered by a technology. Moreover, it is argued [58-61] that individuals with high self-efficacy tend to perform well when conducting a variety of tasks using IT. Pierce et al. [62] found that feelings of self-efficacy encourage individuals to explore and manipulate the environment within which they work and to feel a sense of empowerment. Therefore, it is hypothesized that:

\section{Hypothesis 6-1}

Physician perceptions of Task Technology alignment will be positively impacted by their perceived ability to utilize mHealth.

\section{Hypothesis 6-2}

Physicians perception of their ability to employ mHealth positively impacts utilize mHealth.
Figure 1 presents the conceptual model employed in this study. The next section will discuss how we operationalized this model (Fig. 1).

\section{Methods}

To test the conceptual model (Fig. 1) an observational quasi-experimental one group posttest-only design was employed. The self-reported data was collected by employing a survey and distributed through the internal hospital channels to physicians who adopted iPads for their daily activities. The online survey questionnaire was constructed using indicators already validated in existing research (see Additional file 1). The survey instrument was piloted to ensure content and construct validity. Over 20 medical experts (professionals who work in healthcare delivery and actively utilize mHealth as a part of their daily work related activities) in the US and Ireland participated in the pilot study. Following this, the questionnaire was refined before launching the survey.

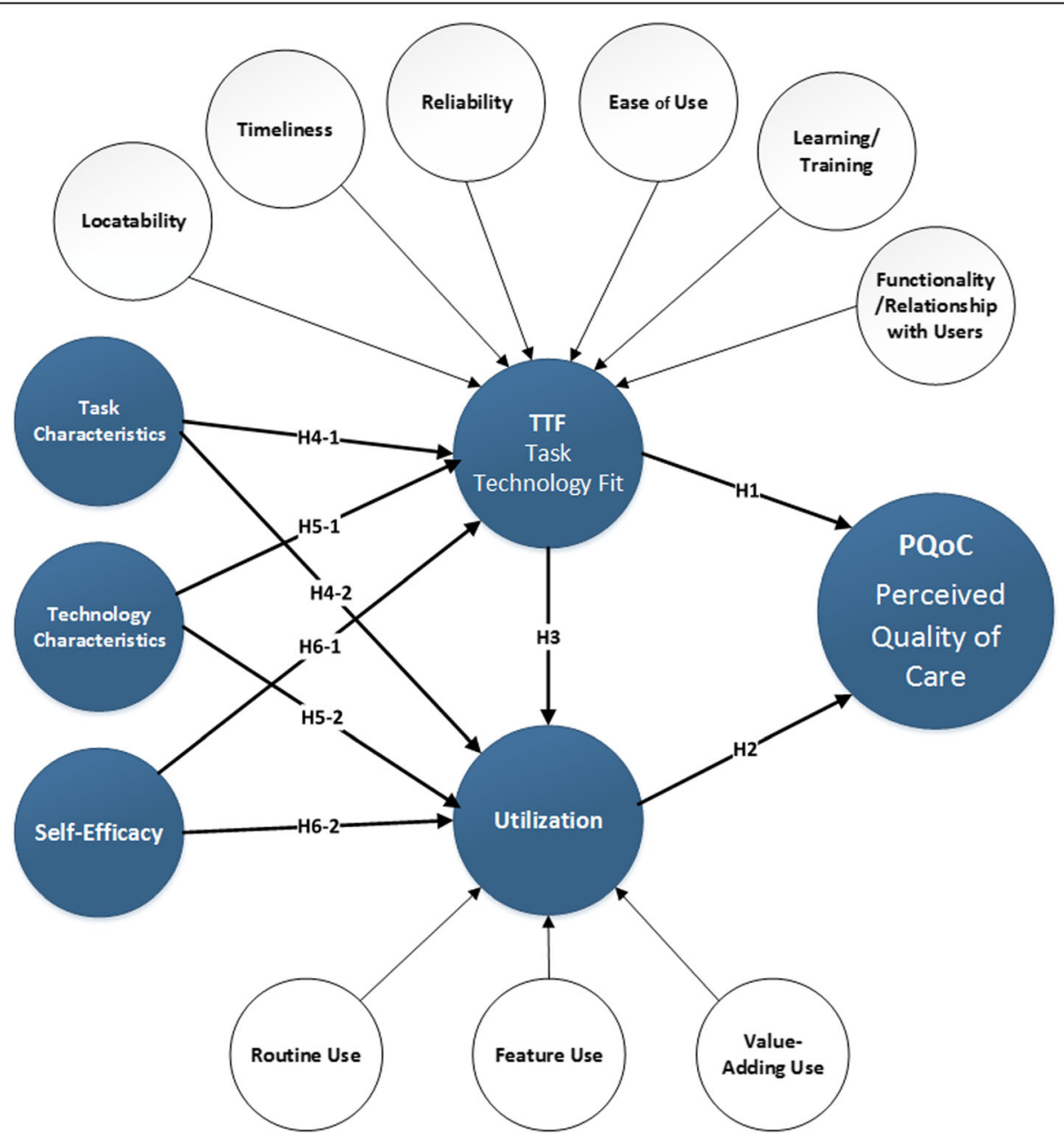

Fig. 1 Conceptual Model 
Data was collected over a four-month period, in 2012, from physicians in an acute care context within The Ottawa Hospital, Ontario, Canada. The Ottawa Hospital $(\mathrm{TOH})$ made over 3000 iPads, incorporating a mobile Electronic Medical Record application, available for use by physicians. $\mathrm{TOH}$ is one hospital spanning three sites (Civic, Riverside, and General Campuses) and has been using mHealth at the point-of-care for numerous years. The survey was distributed via email (an URL link) to physicians in $\mathrm{TOH}$, Canada. No hard copies were distributed to the potential participants since all members of the TOH staff had Internet access.

One major types of bias that is often associated with web surveys is common method variance bias [63]. To overcome the former, several reversed-scored items were used to reduce sign rating problems associated with Likert scales [63]. See Additional file 1 for a description of the items used as part of the questionnaire.

Participants using mHealth within their daily practices for 6 months or more was eligible for the study. A total of 157 responses were obtained from various physicians via the administration of an online survey (871 physicians in total at the time of data collection), yielding a response rate of $18 \%$. Not all of the 871 physicians were available/active during the data collection period of time, which may have impacted the response rate. Noteworthy, it is argued that physicians are often a group with low survey response rates [64]. Nevertheless, 157 responses were cleaned for missing values and 102 complete responses were used for data analysis. While research [65] argues that missing values affects directory of taking the decision, G*Power (version 3.1.2) was used to conduct power analysis and to establish whether the sample size is sufficient. The analysis revealed a power value close to one indicating that the sample size of 102 is sufficient as per Cohen [66]. A key benefit of using Partial Least Square (PLS)- Structural Equation Modeling (SEM) is that it accommodates the use of small sample sizes giving that the ten [10] times rules is met. The 10 times rule depicts that a sample size should be equal to 10 times the largest number of formative indicators used to measure a particular construct, or 10 times the largest number of paths directed at a construct in the model [66]. Our sample size satisfies both requirements.

Structural Equation Modeling (SEM) was used for the hypotheses testing and data analysis. The PLS (SEM) approach, which uses component-based estimation was chosen since it allows simultaneous examination of both the measurement and the structural models. The measurement (outer) model portrays the relationships between a construct and its associated measurement items whereas the structural (inner) model represents direct and indirect unobservable relationships among constructs $[67,68]$. In addition, the PLS approach, in contrast to covariance-based SEM, allows testing of the relationships in the model with less restrictive requirements and relatively small sample sizes. PLS is also considered very appropriate for exploratory studies and for testing theories at earlier stages of development [69], and it is highly suitable for prediction-oriented research [70].

The evaluation process of the PLS path model with mixed formative and reflective constructs involves two steps [70-75]. Step 1 involves the testing of the quality of the measurement (outer) models of firstorder constructs used in the first stage as well as other endogenous and exogenous constructs. After this we assess the appropriateness of the high order constructs. The research model includes two secondorder constructs TTF - task technology fit and Utilization (Fig. 2). We followed the recommendations provided by Becker et al. [76] for repeated indicators, two-stages, and hybrid approaches for estimation hierarchical second-order constructs. As Step 1 was successful and latent constructs were found to be reliable and valid, Step 2, which necessitates the assessment of the structural (inner) model, was conducted [70, 75]. SmartPLS 3.2.6 was employed for the PLS model assessment.

\section{Results}

After excluding 55 responses from the 157 received, 102 surveys were usable for data analysis. This cohort represented 59 males and 43 females, with ages ranging from 18 to 25 years $(n=2) ; 26-40$ years $(n=58) ; 41-55$ years $(n=25) ; \quad 56-65$ years $\quad(n=14) \quad$ and $>65$ years $\quad(n=3)$. Attending Physicians accounted for $53 \%$ of the responses $(n=54)$, with Residents/Fellows completing the remainder of $47 \%$.

The reflective measurement constructs of the research model were assessed according to the established criteria to assess PLS models with reflective constructs (e.g., [67, $70,73])$. The research model includes six first-order reflective constructs of higher-order construct TaskTechnology Fit, three first-order reflective constructs of a second-order construct Utilization, one exogenous reflective construct Self-Efficacy, and one endogenous PQoC (Fig. 1). Hence 10 (ten) reflective measurement models were assessed for reliability and validity. Table 1 demonstrates Individual reliability of the indicators - the magnitudes of all indicators are above this lower limit of 0.707 , with the lowest value of 0.731 and majority of values above 0.8 .

Table 1 illustrates that all manifest items are reliable for all reflective constructs, demonstrating internal consistency reliability since both parameters (i) Cronbach's $\alpha$ and parameter (ii) composite reliability have high values (all values are above 0.752 ), with the 


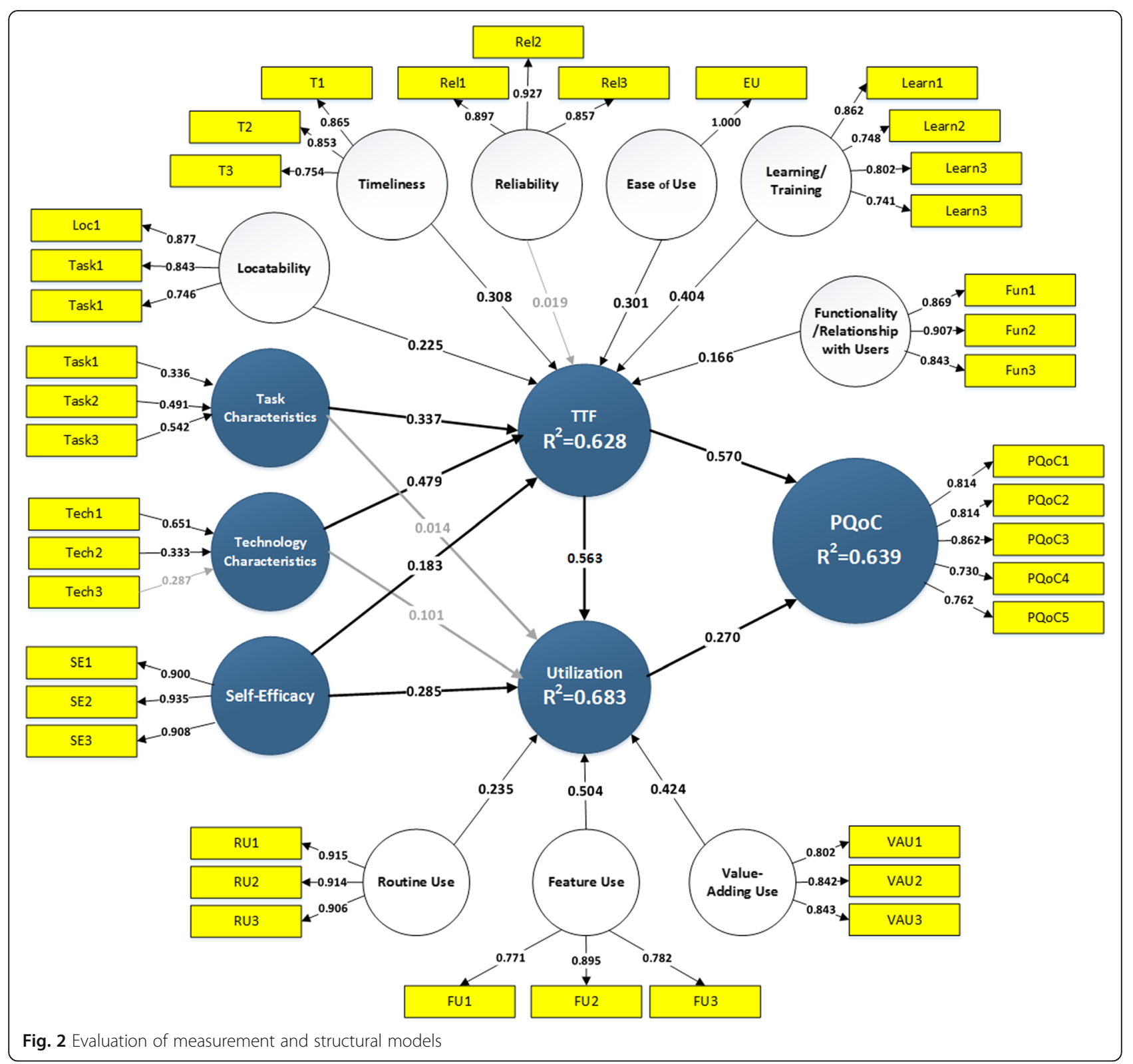

required value being above 0.7 [70]. The validity test of the reflective constructs examines the convergent validity and the discriminant validity. Average Variance Extracted (AVE) for all constructs is higher than 0.5 , which indicates sufficient convergent validity (Table 1).

We tested discriminant validity with three approaches: Fornell-Larker criterion analysis (See Table 2), crossloadings (see Additional file 2), and assessing the heterotrait-monotrait ratio (HTMT) of the correlations (See Table 3).

The results of all tests confirm that the manifest variables (indicators) presented in the research model are reliable and valid.

\section{Assessment of measurement models - formative constructs}

The research model includes two lower order formative constructs: Task Characteristics and Technology Characteristics and two high-order formative contract: TTF and Utilization.

For assessing multicollinearity in the formative measurement model variance inflation factor (VIF) was employed. All VIF indexes were below the critical value of 5 [75] and even lower than the more strict threshold of 3.3 [68], indicating absence of multicollinearity and supporting validations of all indicators (Table 4).

For testing indicator validity, the t-tests of indicator weight significance, accomplished with the SmartPLS 
Table 1 Construct reliability and convergent validity

\begin{tabular}{|c|c|c|c|c|c|c|c|}
\hline \multirow[t]{2}{*}{ Constructs } & \multirow[t]{2}{*}{ Indicator } & \multicolumn{4}{|c|}{ Indicator Reliability/Convergent Validity } & \multicolumn{2}{|c|}{ Internal Consistency Reliability } \\
\hline & & Loading & T-Stat & $p$-value & AVE & Cronbach's a & Composite Reliability \\
\hline \multirow[t]{5}{*}{ Performance (PQoC) } & PQoC1 & 0.815 & 26.58 & 0.00 & 0.636 & 0.856 & 0.897 \\
\hline & PQoC2 & 0.814 & 19.16 & 0.00 & & & \\
\hline & PQoC3 & 0.861 & 23.83 & 0.00 & & & \\
\hline & PQoC4 & 0.731 & 12.11 & 0.00 & & & \\
\hline & PQoC5 & 0.761 & 14.38 & 0.00 & & & \\
\hline \multirow[t]{3}{*}{ Self-efficacy (SE) } & SE1 & 0.900 & 33.41 & 0.00 & 0.836 & 0.902 & 0.939 \\
\hline & SE2 & 0.934 & 34.19 & 0.00 & & & \\
\hline & SE3 & 0.908 & 26.53 & 0.00 & & & \\
\hline \multirow[t]{4}{*}{ Learning/Training (Learn) } & Learn1 & 0.862 & 33.79 & 0.00 & 0.624 & 0.802 & 0.869 \\
\hline & Learn2 & 0.748 & 10.41 & 0.00 & & & \\
\hline & Learn3 & 0.802 & 17.61 & 0.00 & & & \\
\hline & Learn4 & 0.741 & 11.49 & 0.00 & & & \\
\hline \multirow[t]{3}{*}{ Locatability (Loc) } & Loc1 & 0.877 & 25.04 & 0.00 & 0.679 & 0.768 & 0.863 \\
\hline & Loc2 & 0.843 & 13.90 & 0.00 & & & \\
\hline & Loc3 & 0.746 & 9.08 & 0.00 & & & \\
\hline \multirow[t]{3}{*}{ MHealth Reliability (Rel) } & Rel1 & 0.897 & 36.69 & 0.00 & 0.799 & 0.874 & 0.923 \\
\hline & Rel2 & 0.927 & 44.36 & 0.00 & & & \\
\hline & Rel3 & 0.857 & 25.90 & 0.00 & & & \\
\hline \multirow[t]{3}{*}{ Relationship with Users/Functionality (Fun) } & Fun1 & 0.869 & 31.02 & 0.00 & 0.763 & 0.844 & 0.906 \\
\hline & Fun2 & 0.907 & 40.68 & 0.00 & & & \\
\hline & Fun3 & 0.843 & 20.86 & 0.00 & & & \\
\hline \multirow[t]{3}{*}{ Timeliness (T) } & T1 & 0.865 & 20.61 & 0.00 & 0.681 & 0.764 & 0.865 \\
\hline & $\mathrm{T} 2$ & 0.853 & 22.01 & 0.00 & & & \\
\hline & T3 & 0.754 & 9.52 & 0.00 & & & \\
\hline \multirow[t]{3}{*}{ Value-Adding Use (VAU) } & VAU1 & 0.802 & 18.19 & 0.00 & 0.686 & 0.771 & 0.868 \\
\hline & VAU2 & 0.841 & 19.74 & 0.00 & & & \\
\hline & VAU3 & 0.843 & 21.82 & 0.00 & & & \\
\hline \multirow[t]{3}{*}{ Feature Use (FU) } & FU1 & 0.771 & 12.57 & 0.00 & 0.669 & 0.752 & 0.858 \\
\hline & FU2 & 0.895 & 45.54 & 0.00 & & & \\
\hline & FU3 & 0.782 & 10.91 & 0.00 & & & \\
\hline \multirow[t]{3}{*}{ Routine use (RU) } & $\mathrm{RU} 1$ & 0.915 & 42.60 & 0.00 & 0.832 & 0.899 & 0.937 \\
\hline & $\mathrm{RU} 2$ & 0.914 & 43.12 & 0.00 & & & \\
\hline & RU3 & 0.906 & 30.64 & 0.00 & & & \\
\hline
\end{tabular}

program employing the bootstrap method, revealed that almost all endogenous formative latent variables met the requirements of indicator validity. One of the indicators (Tech3) of the Technology Characteristics concept was found not statistically significant with $95 \%$ confidence but significant with $90 \%$. This indicator must be kept in the model since it represents critical dimension of the coordination role of the technology. In second-order construct of TTF the weight of the first-order construct mHealth reliability was not statistically significant.
However, this construct has also been retained in the model due to theoretical consideration.

Routine Use was found as a major Utilization factor $(\gamma=0.540)$. Value-adding Use has a high impact $(\gamma=$ $0.424)$ and is highly important and significant for utilization of mHealth. On the other hand, Feature Use was found although statically significant but the least important factor $(\gamma=0.235)$ in the Utilization construct. Learning/Training was found to be the most important component of Task Technology Fit construct $(\gamma=0.404)$. 
Table 2 Fornell-Larker criterion analysis

\begin{tabular}{|c|c|c|c|c|c|c|c|c|c|c|}
\hline & FU & Learn & LOC & Rel & PQoC & Fun & $R U$ & SE & $\mathrm{T}$ & VAU \\
\hline$\overline{F U}$ & 0.818 & & & & & & & & & \\
\hline Learn & 0.357 & 0.790 & & & & & & & & \\
\hline LoC & 0.465 & 0.307 & 0.824 & & & & & & & \\
\hline Rel & 0.273 & 0.337 & 0.478 & 0.894 & & & & & & \\
\hline PQoC & 0.455 & 0.708 & 0.363 & 0.379 & 0.798 & & & & & \\
\hline Fun & 0.376 & 0.366 & 0.507 & 0.644 & 0.507 & 0.873 & & & & \\
\hline $\mathrm{RU}$ & 0.466 & 0.427 & 0.490 & 0.529 & 0.646 & 0.589 & 0.912 & & & \\
\hline SE & 0.475 & 0.295 & 0.536 & 0.496 & 0.369 & 0.440 & 0.535 & 0.914 & & \\
\hline $\mathrm{T}$ & 0.330 & 0.352 & 0.145 & 0.249 & 0.444 & 0.288 & 0.440 & 0.168 & 0.825 & \\
\hline VAU & 0.464 & 0.632 & 0.409 & 0.276 & 0.613 & 0.359 & 0.571 & 0.485 & 0.456 & 0.828 \\
\hline
\end{tabular}

Timeliness also play a significant role in forming this construct $(\gamma=0.308)$, followed by Ease of use $(\gamma=0.301)$, Locatability $(\gamma=0.225)$, and Relationship with Users/ Functionality with $(\gamma=0.166)$. Accuracy and time criticality (Task3) was the most dominating $(\gamma=0.542)$ in constructing the Task Characteristics construct. Interdependability (Task2) took the second place $(\gamma=0.491)$ and non-routines (Task1) were the weakest source $(\gamma=$ 0.336) in forming this construct. The formation of Technology Characteristics construct Mobility (Tech 1) played the most critical role $(\gamma=0.651)$ followed by Personalization (Tech 2) $(\gamma=0.333)$. Coordination (Tech 3) $(\gamma=0.287)$ was not statistically significant but remained in the model.

For assessing discriminant validity, all correlations of first-order constructs were found to be higher with their second-order constructs than with any other construct in the model. Correlations of formative indicators of two formative constructs were also found higher with their own constructs than with any other construct. Through assessing content validity, construct reliability and validity of the model, it was demonstrated that the measurement models are appropriate and valid. This analysis paved the way for the evaluation of the structural mode.

\section{Assessment of structural model}

The central criterion for evaluating the structural model is the level of explained variance of the dependent constructs. This model explains Perceived Quality of Care Delivery (PQoC), for which the R-square was $63.9 \%$. Also, our model explains $62.8 \%$ of TTF and $68.3 \%$ of Utilization variance (Fig. 2). The variances of all three constructs were explained at the substantial or close to substantial level according to Chin's [74] criteria. $\mathrm{R}^{2}$ values of $0.67,0.33$, or 0.19 for endogenous latent variables are described as substantial, moderate, or weak ( [74] p.323).

All paths coefficients except paths connecting Task and Technology Characteristics with Utilization were found to be highly statistically significant (see Table 5 and Fig. 2).

PQoC was found to be positively affected by TTF (H1 supported with $\beta=0.570$ ) and Utilization (H2 supported

Table 3 Heterotrait-monotrait ratio

\begin{tabular}{|c|c|c|c|c|c|c|c|c|c|c|}
\hline & EU & FU & Learn & LoC & Rel & PQoC & Fun & $R U$ & SE & $\mathrm{T}$ \\
\hline FU & 0.469 & & & & & & & & & \\
\hline Learn & 0.490 & 0.424 & & & & & & & & \\
\hline LOC & 0.487 & 0.618 & 0.359 & & & & & & & \\
\hline Rel & 0.473 & 0.329 & 0.392 & 0.588 & & & & & & \\
\hline PQoC & 0.667 & 0.554 & 0.830 & 0.420 & 0.433 & & & & & \\
\hline Fun & 0.554 & 0.468 & 0.434 & 0.621 & 0.747 & 0.598 & & & & \\
\hline $\mathrm{RU}$ & 0.607 & 0.564 & 0.474 & 0.577 & 0.591 & 0.728 & 0.676 & & & \\
\hline SE & 0.398 & 0.575 & 0.326 & 0.635 & 0.548 & 0.417 & 0.503 & 0.592 & & \\
\hline $\mathrm{T}$ & 0.320 & 0.425 & 0.428 & 0.188 & 0.299 & 0.545 & 0.361 & 0.532 & 0.204 & \\
\hline VAU & 0.376 & 0.588 & 0.759 & 0.512 & 0.331 & 0.745 & 0.448 & 0.685 & 0.584 & 0.593 \\
\hline
\end{tabular}


Table 4 Multicollinearity and indicator-validity tests

\begin{tabular}{|c|c|c|c|c|c|}
\hline Indicator - > Construct & Weight & STD & T-Stat & $P$ Value & VIF \\
\hline Tech1 - > Technology Characteristics & 0.651 & 0.121 & 5.396 & 0.000 & 1.251 \\
\hline Tech2 - > Technology Characteristics & 0.333 & 0.167 & 1.997 & 0.046 & 1.367 \\
\hline Tech3 - > Technology Characteristics & 0.287 & 0.165 & 1.742 & 0.082 & 1.576 \\
\hline Task1 - > Task Characteristics & 0.336 & 0.163 & 2.067 & 0.039 & 1.149 \\
\hline Task2 - > Task Characteristics & 0.491 & 0.168 & 2.926 & 0.003 & 1.207 \\
\hline Task3 - > Task Characteristics & 0.542 & 0.181 & 2.987 & 0.003 & 1.120 \\
\hline Ease of use $->$ TTF & 0.301 & 0.075 & 3.991 & 0.000 & 1.604 \\
\hline Learning/Training - > TTF & 0.404 & 0.080 & 5.027 & 0.000 & 1.387 \\
\hline Locatability - > TTF & 0.225 & 0.076 & 2.971 & 0.003 & 1.502 \\
\hline MHEALTH Reliability- > TTF & 0.019 & 0.085 & 0.228 & 0.820 & 1.850 \\
\hline Relationship with Users/Functionality- > TTF & 0.166 & 0.083 & 2.001 & 0.045 & 2.050 \\
\hline Timeliness - > TTF & 0.308 & 0.097 & 3.190 & 0.001 & 1.197 \\
\hline Feature Use - > Utilization & 0.235 & 0.093 & 2.518 & 0.012 & 1.379 \\
\hline Routine use - > Utilization & 0.540 & 0.097 & 5.565 & 0.000 & 1.607 \\
\hline Value-Adding Use - > Utilization & 0.424 & 0.111 & 3.807 & 0.000 & 1.603 \\
\hline
\end{tabular}

with $\beta=0.270$ ). TTF affects Utilization (H3 supported with $\beta=0.563$ ). TTF was found to be positively affected by Task Characteristics (H4-1 supported with $\beta=$ 0.335), Technology Characteristics (H5-1 supported with $\beta=0.479$ ), and Self-efficacy (H6-1 supported with $\beta=0.184$ ), and Internal Knowledge (H5 supported with $\beta=0.379$ ). In addition to TTF, we found that selfefficacy also affects Utilization (H6-2 supported with $\beta=0.295$ ), while task and technology characteristics do not affect Utilization (H4-2 and H5-2 not supported).

In addition to path coefficients that represent direct effects we assessed indirect and total effects of the constructs. Changes in R-square were explored to investigate the substantive impact of each independent construct on the dependent construct, carrying out the effect size technique by re-running PLS estimations, excluding one explaining latent construct in each run. Chin [74] proposed an effect size $f^{2}$ categorization of PLS constructs similar to Cohen's implementation for multiple regression: small $\left(f^{2}=0.02\right)$, medium $\left(f^{2}=0.15\right)$, and large $\left(f^{2}=0.35\right)$. TTF has a large effect on both $P Q o C$ and Utilization (with $\mathrm{f}^{2}=0.351$ and $\mathrm{f}^{2}=0.372$ accordingly). While the effect of Utilization on PQoC is small $\left(f^{2}=0.079\right)$. There is a large effect of Technology Characteristics on TTF $\left(f^{2}=0.444\right)$, while effect of Task Characteristics is medium $\left(f^{2}=0.243\right)$ and Self-efficacy has a small effect on TTF $\left(f^{2}=0.072\right)$ and medium on Utilization $\left(f^{2}=0.192\right)$.

Table 5 Path coefficients significance test. Direct, indirect, and total effects

\begin{tabular}{|c|c|c|c|c|c|c|c|}
\hline & Path (Direct) & T-Stat & $P$ value & Indirect & Total & T-Stat & $P$ Value \\
\hline Self-Efficacy - > TTF (H6-1) [+] & 0.183 & 2.261 & 0.024 & 0.000 & 0.183 & 2.261 & 0.024 \\
\hline Self-Efficacy - > Utilization (H6-2) [+] & 0.285 & 3.631 & 0.000 & 0.103 & 0.388 & 4.692 & 0.000 \\
\hline TTF - > PQoC (H1) [+] & 0.570 & 6.194 & 0.000 & 0.152 & 0.722 & 12.658 & 0.000 \\
\hline TTF - > Utilization (H3) [+] & 0.563 & 5.203 & 0.000 & 0.000 & 0.563 & 5.203 & 0.000 \\
\hline Task Characteristics - > TTF (H4-1) [+] & 0.337 & 3.718 & 0.000 & 0.000 & 0.337 & 3.718 & 0.000 \\
\hline Task Characteristics - > Utilization (H4-2) [-] & 0.014 & 0.180 & 0.858 & 0.190 & 0.204 & 2.379 & 0.017 \\
\hline Tech Characteristics - > TTF (H5-1) [+] & 0.479 & 5.581 & 0.000 & 0.000 & 0.479 & 5.581 & 0.000 \\
\hline Tech Characteristics - > Utilization (H5-2) [-] & 0.101 & 1.072 & 0.284 & 0.270 & 0.371 & 4.380 & 0.000 \\
\hline Utilization - > PQoC (H2) [+] & 0.270 & 2.790 & 0.005 & 0.000 & 0.270 & 2.790 & 0.005 \\
\hline Self-Efficacy - > PQoC_ & 0.000 & & & 0.209 & 0.209 & 3.440 & 0.001 \\
\hline Task Characteristics - > PQoC_ & 0.000 & & & 0.247 & 0.247 & 3.526 & 0.000 \\
\hline Technology Characteristics - > PQoC & 0.000 & & & 0.373 & 0.373 & 5.302 & 0.000 \\
\hline
\end{tabular}


For the evaluation of the predictive relevance of the structural model, the Stone and Geisser test was performed using the blindfolding procedure. $Q^{2}$ reflects an index of goodness of reconstruction by model and parameter estimations. A positive $Q^{2}>0$ provides evidence that the omitted observations (from blindfolding) were well-reconstructed and that predictive relevance is achieved, while a negative $Q^{2}$ reflects absence of predictive relevance. All values of $Q^{2}$ were greater than zero, indicating predictive relevance for the endogenous constructs of the research model. Table 6 shows that the $Q^{2}$ effect size for the relationships of TTF with PQoC and Utilisation can be considered as close to medium prediction relevance. Predictive relevance of Technology characteristics with respect to TTF can be considered as between small and medium while the rest of relationships have small $Q^{2}$ effect size.

\section{Discussion}

While the TTF theory has been studied in health domains $[20,77]$ and even variant model has been suggested such as inclusion of self-efficacy in the model [78, 79] and feed-forward chain in the TTF theory [35], a dearth of research focuses on the impact on PQoC [20]. Towards addressing this shortcoming in existing research, this study examines the impact of mHealth on the PQoC in a post-adoptive scenario. The conceptual model was developed and empirically tested (Fig. 2). The model explains $64 \%$ of the PQoC. Furthermore, it also explains 63 and $68 \%$ respectively of the endogenous constructs (TTF and utilization). TTF was found to be the dominant construct in explaining the variance of PQoC. We can infer that in a post-adoptive scenario, TTF becomes fundamental (and a very important mediator) for PQoC. Realizing how an organization can improve TTF will lead to better PQoC.

A systematic review [80] on mHealth adoption by healthcare professionals found that perceived usefulness and ease of use, design and technical concerns, cost, time, privacy and security issues, familiarity with the technology, risk-benefit assessment, and interaction with others (colleagues, patients, and management) are the main factors to providers' adoption behavior. Our research corroborates these findings. However, towards

Table 6 The effect size $Q^{2}$ predictive relevance test

\begin{tabular}{llll}
\hline & PQoC & TTF & Utilisation \\
\hline Task & 0.00 & 0.03 & -0.01 \\
Technology & 0.01 & 0.10 & 0.00 \\
Self-Efficacy & -0.01 & 0.03 & 0.06 \\
Utilisation & 0.01 & & \\
TTF & 0.12 & & 0.12 \\
\hline
\end{tabular}

understanding how to improve TTF, our model tested the main components of TTF and found that physicians should keep investing in learning and training, regardless of the stages of technology adoption. Learning and Training was found to be the most critical factor in the formation of TTF. Training and implementation management, as part of the Normalisation Process Theory (NPT), is argued [81] to promote the successful implementation and integration of interventions into routine work. Timeliness and Ease of Use were found to be the second and third factors in the formation of TTF. The medical domain is one which is continuously evolving, necessitating physicians to continuously learn. By utilizing mHealth to keep up to date with the latest clinical/ medical protocols, physicians are continuously striving to improve quality of care.

Research confirms that self-efficacy plays an important role for IT utilization (cf. $[55,56,82,83]$ ). In a postadoptive scenario, the findings reveal that self-efficacy is critical for utilization, and this construct has the highest direct and total effect on utilization. Interpreting this finding, there is an ongoing requirement to continuously enhance an individual's skillset for using mHealth. The findings reveal that self-efficacy has a small effect on TTF, which is unsurprising given the fact that the mHealth is already embedded in a physicians' work practices. In the formation of TTF, the findings further reveal that technological characteristics dominate followed by task characteristics.

In the conceptual model (Fig. 2), the alignment between task and technology (TTF) has an impact upon use. Interestingly, our findings reveal that (1) the technological characteristics of mHealth and (2) healthcare physician's work practices have no direct impact upon utilization. Towards explaining these findings, one should consider the context of the study. Data was gathered at a post-adoptive stage, meaning that mHealth had been continuously used over an extended period of time resulting in mHealth being embedded in physicians' work practices. It was found that there is no direct impact of task characteristics and technology characteristics on mHealth utilization, although we hypothesized these impacts. However, both these constructs have significant indirect effects (TTF is a mediator) and total effects on utilization. Therefore, at the most advanced stages of utilization direct impacts are not relevant, but TTF becomes a very important mediator.

A user's behavior can range from stagnation in utilizing IT features to total integration of the IT in his/her work domain [84-86]. Therefore, it is important that the features/functionalities of mHealth can be adapted easily to reflect the true but constant changing working nature of physicians to complete any given task within hospitals. MHealth containing electronic pharmacopoeias (i.e. 
drug information), medical calculations, guideline information and administrative tasks have been identified as the most useful resources by physicians, nurses and other clinical staff [87].

In the context of understanding utilization and its constituent parts, this article decomposes utilization into routine, feature, and value-adding use; the three of which have not been collectively examined in explaining utilization. By breaking utilization into these three constituent parts, we were able to identify that routine use and value-adding use are the major utilization factors for mHealth when delivering healthcare services at the point of care. At the earliest stages of adoption routine use can be very critical. However, at a post-adoption stage, routine use, while statistically significant, is the least important factor in mHealth utilization; feature use takes the leading role. An explanation for this is that as system usage becomes repetitive and habitual, routine use emerges. This confirms existing research [53]. In the context of value-adding use, physicians take advantage of the current artefact by exploring features which they as individuals are less familiar with. This potentially enables mHealth to be used by physicians in novel ways/for unanticipated emerging patient problems. Therefore, in post-adoptive scenarios physicians may employ different features to cope with changing working requirements.

\section{Conclusion}

This article answers calls for the development of a specific mHealth evaluation framework which is scant in existing literature [8-11]. In meeting this request, we have also addressed the dearth of research examining mHealth in a post-adoptive scenario and its impact upon Perceived Quality of Care Delivery (PQoC).

MHealth presents healthcare organisations with a significant amount of opportunity which benefits healthcare professionals and patients alike. This study informs hospitals and software vendors as to the performance of mHealth by clearly demonstrating that physicians using mHealth at the point-of-care enhances their PQoC a patient receives. As the availability of mHealth continues to increase, we call that all mHealth should be reviewed by clinical experts in order to safeguard the quality of care patients receive.

This study also contributes to the practitioner community by highlighting the importance of adapting mHealth to adhere to users work practices, without unnecessary disruption to the use of the service. Changes in work practices within healthcare environments are often dictated by external forces (e.g. pharmaceutical society introduces new guidelines for dispensing drugs). For PQoC to remain constant, it is imperative that mHealth continuously evolves and adapts to changing work practices and that mHealth be designed with work practices in mind. Indeed, our findings reveal that once technology is embedded, technology characteristics are a secondary consideration for physicians.

Although this research achieved its objective, the results of this study should be interpreted in the context of its limitations. First, this model was examined from a healthcare physician perspective. While a healthcare physician population was appropriate for this study, the conceptual model (see Fig. 1) could be tested across a wide cohort of medical professions (e.g. nurses, physiotherapists, dieticians and, pharmacists). Such context extensions are argued ([88]) p.103) to be "part of on-going efforts to provide generalised measures of TTF constructs". Additionally, healthcare services are often delivered across different levels (e.g. primary, secondary and territory) and scenarios (e.g. preventive care, urgent care, emergency care, home health, and long-term care) ( [89] p.66). As a result, we further urge future research to also consider these domains. Moreover, individuals use mobile technology, especially smartphones, for both hedonic and utilitarian purposes [90]. Building from this, future research should examine medical professionals who use smartphones which are consumed for both work and personal purposes. Although rich data was obtained from participants in the study to develop and validate the conceptual model, future research could conduct similar empirical work with a larger study population. This will further validate the research model.

\section{Supplementary information}

Supplementary information accompanies this paper at https://doi.org/10. 1186/s12911-020-1049-8.

\section{Additional file 1.}

Additional file 2 .

\section{Abbreviations}

H: Hypothesis; IT: Information Technology; MHealth: Mobile health; PLS: Partial Least Squares; PQoC: Perceived quality of care delivery; SEM: Structural Equation Modeling; TTF: Task-technology fit

\section{Acknowledgements \\ Not applicable.}

\section{Authors' contributions}

YOC and POR designed and supervised the study. PA and YOC analysed the data. YOC, PA, and POR drafted the manuscript. All authors read and approved the final manuscript.

\section{Funding}

Not applicable.

Availability of data and materials

All data generated or analyzed during this study are included in this published article [and its supplementary information files]. 


\section{Ethics approval and consent to participate}

This study was reviewed by the research ethics board at the Ottawa Hospital, Canada. Organizational level written consent (by email) was provided for this study.

\section{Consent for publication}

Not applicable; no identifying information on any individual's data is presented in this manuscript.

\section{Competing interests}

The authors declare that they have no competing interests.

\section{Author details}

'Department of Business Information Systems, University College Cork, $\mathrm{O}^{\prime}$ Rahilly Building, Cork, Ireland. ${ }^{2}$ Telfer School of Management, University of Ottawa, Ottawa, Canada.

\section{Received: 15 October 2019 Accepted: 11 February 2020}

Published online: 27 February 2020

\section{References}

1. Lee $\mathrm{J}-\mathrm{H}$. Future of the smartphone for patients and healthcare providers Healthcare Inform Res. 2016;22(1):1-2.

2. Sherer $\mathrm{S}$, Meyerhoefer $C$, Levick D. Challenges to aligning coordination technology with organizations, people, and processes in healthcare. In: Proceedings of the 50th Hawaii international conference on system sciences; 2017

3. Seyedi H, Mohebbifar R, Rafiei S. Quality management system and its role in the quality maturity of training hospitals. J Client-Centered Nursing Care. 2019;5(2):113-22.

4. Kohli R, Tan SS-L. Electronic health records: how can IS researchers contribute to transforming healthcare? MIS Q. 2016:40(3):553-73.

5. Sarre S, Maben J, Griffiths P, Chable R, Robert G. The 10-year impact of a ward-level quality improvement intervention in acute hospitals: a multiple methods study. Southampton: NIHR Journals Library, Health Services and Delivery Research. 2019;7(28)

6. Abu-Dalbouh $\mathrm{H}$. A proposed mhealth model for improving the quality care in hospitals. Res J Appl Sci Eng Technol. 2014;7(7):1401-5.

7. Rahurkar S, Vest JR, Menachemi N. Despite the spread of health information exchange, there is little evidence of its impact on cost, use, and quality of care. Health Aff. 2015;34(3):477-83.

8. Kumar S, Nilsen WJ, Abernethy A, Atienza A, Patrick K, Pavel M, et al. Mobile health technology evaluation: the mHealth evidence workshop. Am J Prev Med. 2013;45(2):228-36.

9. Hall CS, Fottrell E, Wilkinson S, Byass P. Assessing the impact of mHealth interventions in low-and middle-income countries-what has been shown to work? Glob Health Action. 2014;7:25606.

10. Dick S, O'Connor Y, Heavin C. Approaches to Mobile health evaluation: a comparative study a mHealth evaluation comparison study. Inf Syst Manag. 2019;37:1-18.

11. Boudreaux ED, Waring ME, Hayes RB, Sadasivam RS, Mullen S, Pagoto S. Evaluating and selecting mobile health apps: strategies for healthcare providers and healthcare organizations. Transl Behav Med. 2014;4(4):363-71.

12. Keikhosrokiani P, Zakaria N, Mustaffa N, Venkat I. Study of the effective factors in Mobile health-care success: sociotechnical perspective. In: mHealth Multidisciplinary Verticals; 2014. p. 237.

13. Anglada-Martinez H, Riu-Viladoms G, Martin-Conde M, Rovira-Illamola M, Sotoca-Momblona J, Codina-Jane C. Does mHealth increase adherence to medication? Results of a systematic review. Int J Clin Pract. 2015;69(1):9-32.

14. Franz-Vasdeki J, Pratt BA, Newsome M, Germann S. Taking mHealth solutions to scale: enabling environments and successful implementation. J Mobile Technol Med. 2015:4(1):35-8.

15. Hamine S, Gerth-Guyette E, Faulx D, Green BB, Ginsburg AS. Impact of mHealth chronic disease management on treatment adherence and patient outcomes: a systematic review. J Med Internet Res. 2015;17(2):e52.

16. Turner T, Spruijt-Metz D, Wen C, Hingle M. Prevention and treatment of pediatric obesity using mobile and wireless technologies: a systematic review. Pediatr Obes. 2015;10:403-9.

17. Hsieh JP-A, Zmud R. Understanding post-Adtopive usage behaviors: a twodimensional view. DIGIT 2006 proceedings; 2006.
18. O'Connor Y, O'Reilly P. Examining the infusion of mobile technology by healthcare practitioners in a hospital setting. Inf Syst Front. 2018;20(6):1297-317.

19. Goodhue DL, Thompson RL. Task-technology fit and individual performance. MIS Q. 1995:19(2):213-36.

20. Serrano $\mathrm{Cl}$, Karahanna E. The compensatory interaction between user Capabiities and technology capabilities in influencing task performance: an empirical assessment in telemedicine consultations. MIS Q. 2016;40(3):597-621.

21. Sundin P, Callan J, Mehta K. Why do entrepreneurial mHealth ventures in the developing world fail to scale? J Med Eng Technol. 2016;40(7-8):1-14.

22. Varshney U. Mobile health: four emerging themes of research. Decis Support Syst. 2014;66(0):20-35.

23. Sun H, Fang Y, Zou HM. Choosing a fit technology: understanding mindfulness in technology adoption and continuance. J Assoc Inf Syst. 2016;17(6):377.

24. Bhuyan S, Kim H, Isehunwa OO, Kumar N, Bhatt J, Wyant DK, et al. Privacy and security issues in mobile health: current research and future directions. In: Health policy and technology; 2017.

25. Restuccia JD, Cohen AB, Horwitt JN, Shwartz M. Hospital implementation of health information technology and quality of care: are they related? BMC Med Inform Decis Mak. 2012;12(1):109.

26. Miah SJ, Gammack J, Hasan N. Extending the framework for Mobile health information systems research: a content analysis. Inf Syst. 2017;69:1-24.

27. Cook VE, Ellis AK, Hildebrand KJ. Mobile health applications in clinical practice: pearls, pitfalls, and key considerations. Ann Allergy Asthma Immunol. 2016;117(2):143-9.

28. Donabedian A. Commentary on some studies of the quality of care. Health Care Financ Rev. 1987:SUPPL:75-85.

29. Chang JT, Hays RD, Shekelle PG, MacLean CH, Solomon DH, Reuben DB, et al. Patients' global ratings of their health care are not associated with the technical quality of their care. Ann Intern Med. 2006;144(9):665-72.

30. Hanefeld J, Powell-Jackson T, Balabanova D. Understanding and measuring quality of care: dealing with complexity. Bull World Health Organ. 2017; 95(5):368.

31. Scott Morton MS. The corporation of the 1990s. Information technology and organizational transformation; 1991.

32. Chatfield AT, Yetton P. Strategic payoff from EDI as a function of EDI embeddedness. J Manag Inf Syst. 2000;16(4):195-224.

33. Bensaou M, Venkatraman N. Inter-organizational relationships and information technology: a conceptual synthesis and a research framework. Eur J Inf Syst. 1996:5(2):84-91.

34. Doran D, Haynes BR, Estabrooks CA, Kushniruk A, Dubrowski A, Bajnok I, et al. The role of organizational context and individual nurse characteristics in explaining variation in use of information technologies in evidence based practice. Implement Sci. 2012;7(1):122.

35. Chiasson M, Kelley H, Downey A. Understanding task-performance chain feed-forward and feedback relationships in E-health. AIS Transact HumanComput Interact. 2015;7(3):167-90.

36. Furneaux B. Task-technology fit theory: a survey and synopsis of the literature. In: Dwivedi Y., Wade M., Schneberger S. (eds) Information Systems Theory. Integrated Series in Information Systems, vol 28. New York: Springer; 2012.

37. Kutney-Lee A, Sloane DM, Bowles KH, Burns LR, Aiken LH. Electronic health record adoption and nurse reports of usability and quality of care: the role of work environment. Appl Clin Inform. 2019;10(01):129-39.

38. Abraham D. A grounded theory for the impacts of ubiquitous information systems (IS) access on task performance. Austin: Austin Mobility Roundtable; 2004.

39. Basole RC. The value and impact of mobile information and communication technologies. Atlanta: Proceedings of the 2004 International Federation of Automatic Control Symposium; 2004; 2004.

40. Hsiao J-L, Chen R-F. An investigation on task-technology fit of Mobile nursing information Systems for Nursing Performance. Comput Inform Nurs. 2012;30(5):265-73.

41. Rossi M, Tuunainen VK, Pesonen M. Mobile technology in field customer service: big improvements with small changes. Bus Process Manag J. 2007; 13(6):853-65.

42. Kim H-W, Chan HC, Lee S-H. A user commitment approach to information systems infusion: PACIS 2012 Proceedings; 2012. Paper 101. Ho Chi Minh City, Vietnam.

43. Thatcher JB, Wright RT, Sun H, Zagenczyk TJ, Klein R. Mindfulness in information technology use: definitions, distinctions, and a new measure. MIS Q. 2018:42(3):831-47. 
44. McLean E, Sedera D, Tan F. Reconceptualizing system use for contemporary information systems. In: PACIS 2011 proceedings; 2011. p. 130.

45. Oakley R, Palvia P. A study of the impact of Mobile self-efficacy and emotional attachment on Mobile device infusion. Seattle: AMCIS 2012; 2012. Paper 15.

46. Limayem M, Hirt SG. Force of habit and information systems usage: theory and initial validation. J Assoc Inf Syst. 2003:4(1):Article 3.

47. Prgomet M, Georgiou A, Westbrook Jl. The impact of mobile handheld technology on hospital physicians' work practices and patient care: a systematic review. J Am Med Inform Assoc. 2009;16(6):792-801.

48. Bell H, Garfield S, Khosla S, Patel C, Franklin BD. Mixed methods study of medication-related decision support alerts experienced during electronic prescribing for inpatients at an English hospital. Eur J Hosp Pharm. 2019; 26(6):318-22.

49. Mans R, van der Aalst W, Russell N, Bakker P. Implementation of a healthcare process in four different workflow systems. Technical report. Eindhoven: Technische Universiteit Eindhoven; 2009.

50. Han S, Harkke V, Mustonen P, Seppanen M, Kallio M. Mobilizing medical information and knowledge: some insights from a survey. In: ECIS 2004 proceedings; 2004. p. Paper 69

51. Venkatesh V, Brown SA, Maruping LM, Bala H. Predicting different conceptualizations of system use: the competing roles of behavioral intention, facilitating conditions, and behavioral expectation. MIS Q. 2008; 32(3):483-502.

52. Agarwal $R$, Venkatesh $V$. Assessing a firm's web presence: a heuristic evaluation procedure for the measurement of usability. Inf Syst Res. 2002; 13(2):168-86.

53. Cooper RB, Zmud RW. Information technology implementation research: a technological diffusion approach. Manag Sci. 1990;36(2):123-39.

54. Lewis W, Agarwal R, Sambamurthy V. Sources of influence on beliefs about information technology use: an empirical study of knowledge workers. MIS Q. 2003;27(4):657-78.

55. Tsai M-F, Hung S-Y, Yu W-J, Chen C, Yen DC. Understanding physicians' adoption of electronic medical records: healthcare technology self-efficacy, service level and risk perspectives. Comput Stand Interfaces. 2019; 66(103342).

56. Compeau DR, Higgins CA. Computer self-efficacy: development of a measure and initial test. MIS Q. 1995;19(2):189-211.

57. Shaw N, Manwani S. Extending feature usage: a study of the post-adoption of electronic medical records CIS 2011 proceedings; 2011. p. Paper 125.

58. Bandura A. Self-efficacy: toward a unifying theory of behavioral change. Psychol Rev. 1977;84(2):191-215.

59. Stajkovic AD, Luthans F. Self-efficacy and work-related performance: a metaanalysis. Psychol Bull. 1988;124(2):240-61.

60. Vannatta R, Beyerbach B, Walsh C. From teaching technology to using technology to enhance student learning: preservice teachers' changing perceptions of technology infusion. J Technol Teach Educ. 2001;9(1): $105-27$.

61. Pongpattrachai D, Cragg P, Fisher R. Spreadsheet infusion in small audit firms in Thailand. In: Americas conference on information systems Paper; 2009. p. 432.

62. Pierce JL, Kostova T, Dirks KT. The state of psychological ownership: integrating and extending a century of research. Rev Gen Psychol. 2003;7(1):84-107.

63. Podsakoff PM, MacKenzie SB, Lee J-Y, Podsakoff NP. Common method biases in behavioral research: a critical review of the literature and recommended remedies. J Appl Psychol. 2003;88(5):879.

64. Cunningham CT, Quan H, Hemmelgarn B, Noseworthy T, Beck CA, Dixon E, et al. Exploring physician specialist response rates to web-based surveys. BMC Med Res Methodol. 2015;15(1):32.

65. Al-Janabi S, Alkaim AF. A nifty collaborative analysis to predicting a novel tool (DRFLLS) for missing values estimation. Soft Comput. 2019;24:555-69.

66. Cohen J. Statistical power analysis for the behavioral sciences ed. Hillsdale: Lawrence Erlbaum Associates, NJ; 1988.

67. Tenenhaus M, Vinzi VE, Chatelin YM, Lauro C. PLS path modeling. Comput Stat Data Anal. 2005;48(1):159-205.

68. Diamantopoulos A, Siguaw JA. Formative versus reflective indicators in organizational measure development: a comparison and empirical illustration. Br J Manag. 2006;17(4):263-82.

69. Fornell C, Bookstein FL. Two structural equation models: LISREL and PLS applied to consumer exit-voice theory. J Market Res. 1982;19(4):440-52.

70. Henseler J, Ringle CM, Sinkovics RR. The use of partial least squares path modeling in international marketing. Adv Int Mark. 2009;20(2009):277-319.
71. Andreev P, Heart T, Maoz H, Pliskin N. Validating formative partial least squares (PLS) models: methodological review and empirical illustration. Phoenix: ICIS 2009 Proceedings; 2009. p. 193.

72. Chatelin YM, Vinzi VE, Tenenhaus M. State-of-art on PLS path modeling through the available software. In: Les cahiers de Recherche. Paris: Groupe HEC; 2002. p. 764.

73. Gefen D, Straub DW, Boudreau M-C. Structural equation modeling and regression: guidelines for research and practice. CAIS. 2000;4(7):1-70.

74. Chin WW. The partial least squares approach to structural equation modeling. In: G.E M, editor. Modern methods for business research Mahwah. New Jersey: Lawrence Erlbaum Associates; 1998. p. 295-336.

75. Hair JF Jr, Hult GTM, Ringle C, Sarstedt M. A primer on partial least squares structural equation modeling (PLS-SEM): sage publications; 2016.

76. Becker J-M, Klein K, Wetzels M. Hierarchical latent variable models in PLSSEM: guidelines for using reflective-formative type models. Long Range Plann. 2012;45(5):359-94

77. Ali SB, Romero J, Morrison K, Hafeez B, Ancker JS. Focus section health IT usability: applying a task-technology fit model to adapt an electronic patient portal for patient work. Appl Clin Inform. 2018;9(01):174-84.

78. Dishaw M, Strong D, Bandy DB. Extending the task-technology fit model with self-efficacy constructs. In: AMCIS 2002 proceedings; 2002. p. 143.

79. Lin T-C, Huang C-C. Understanding knowledge management system usage antecedents: an integration of social cognitive theory and task technology fit. Inf Manage. 2008;45(6):410-7.

80. Gagnon M-P, Ngangue P, Payne-Gagnon J, Desmartis M. M-health adoption by healthcare professionals: a systematic review. J Am Med Inform Assoc. 2015;23(1):212-20

81. Murray E, Treweek S, Pope C, MacFarlane A, Ballini L, Dowrick C, et al. Normalisation process theory: a framework for developing, evaluating and implementing complex interventions. BMC Med. 2010;8(1):63.

82. Beaudry A, Pinsonneault A. Advancing the theory of infusion: an appropriation model of the infusion process. Paris: Cahier du GreSI M, Canada; 1999.

83. Igbaria M, livari J. The effects of self-efficacy on computer usage. Omega. 1995;23(6):587-605.

84. Agarwal R, Karahanna E. Time flies when you're having fun: cognitive absorption and beliefs about information technology usage. MIS Q. 2000;24: 665-94.

85. Jain $\mathrm{V}$, Kanungo $\mathrm{S}$. IS-enabled performance improvement at the individual level: evidence of complementarity. In: Proceedings of the 2006 ACM SIGMIS CPR conference on computer personnel research: forty four years of computer personnel research: achievements, challenges and the future. Claremont. 1125181: ACM; 2006. p. 25-33.

86. Sun $\mathrm{H}$. Understanding user revisions when using information system features: adaptive system use and triggers. MIS Q. 2012;36(2):453-78.

87. Honeybourne C, Sutton S, Ward L. Knowledge in the palm of your hands: PDAs in the clinical setting. Health Info Libr J. 2006;23(1):51-9.

88. Strong DM, Dishaw MT, Bandy DB. Extending task technology fit with computer self-efficacy. ACM SIGMIS Database. 2006;37(2-3):96-107.

89. Varshney U. A model for improving quality of decisions in mobile health. Decis Support Syst. 2014;62:66-77.

90. Wakefield RL, Whitten D. Mobile computing: a user study on hedonic/ utilitarian mobile device usage. Eur J Inf Syst. 2006;15(3):292-300.

\section{Publisher's Note}

Springer Nature remains neutral with regard to jurisdictional claims in published maps and institutional affiliations.

\section{Ready to submit your research? Choose BMC and benefit from:}

- fast, convenient online submission

- thorough peer review by experienced researchers in your field

- rapid publication on acceptance

- support for research data, including large and complex data types

- gold Open Access which fosters wider collaboration and increased citations

- maximum visibility for your research: over $100 \mathrm{M}$ website views per year

At BMC, research is always in progress.

Learn more biomedcentral.com/submissions 